\title{
Two-pronged tactics for malaria control
}

\begin{abstract}
Most existing and investigational drugs for malaria are targeted at the stages of the Plasmodium spp. parasite life cycle in red blood cells, which underlie the disease manifestations. Targeting the earlierstage exo-erythocytic forms, which multiply in the host liver following infection before emerging into the bloodstream, could be prophylactic but developing high-throughput assays for such activity has been more technically challenging than for blood-stage activity. Writing in Science, an academia-industry consortium describes the use of a high-content imaging assay of liverstage activity to identify a new class of compounds that confer complete protection against parasite challenge in rodent models of malaria, as well as showing potent in vivo activity against blood-stage parasites.

In vitro, only $\sim 1 \%$ of liver cells become infected by malaria parasites at the sporozoite stage of the life cycle, and so to provide the throughput necessary for screening thousands of compounds the authors refined an in vitro assay with Plasmodium yoelii sporozoites to identify infected cells through high-content imaging analysis. They then used this assay to screen a set of $>4,000$ commercially available compounds that had previously been shown to have blood-stage activity.

Based on an analysis of the structures of compounds that possessed
\end{abstract}

both liver-stage and blood-stage activity, a cluster of compounds containing an imidazolopiperazine scaffold was selected for further investigation, which was considered to be attractive because it is structurally unrelated to existing drugs and is chemically tractable. By synthesizing $>1,200$ imidazolopiperazine derivatives and optimizing their potency against blood-stage parasites, a compound - known as GNF179 - was identified that also possessed suitable pharmacokinetic properties for oral testing in animal models of malaria.

In mice infected with Plasmodium berghei, GNF179 lowered parasitaemia levels by $99.7 \%$ after the administration of a single $100 \mathrm{mg}$ per $\mathrm{kg}$ oral dose, and prolonged the survival of mice by an average of 19 days, compared with 12.5 days and 7 days for the existing antimalarial drugs chloroquine and artesunate, respectively. Furthermore, GNF179 provided complete protection against a $P$. berghei sporozoite challenge after a single $15 \mathrm{mg}$ per $\mathrm{kg}$ oral dose.

Initial studies of the mechanism of action of GNF179 indicated that it was distinct from existing drugs, and also that its target might be a protein (encoded by a parasite gene known as $p f c a r 1$ ) that has been tentatively linked to protein folding in the endoplasmic reticulum, which would presumably be important in both the liver and blood stages of parasite infection. Given that antimalarials with novel mechanisms of action are desirable because they could be less likely to show crossresistance with existing drugs, this study provides a valuable starting point for developing more effective drugs that might be used not only to treat infected patients but also to provide protection against infection and reduce transmission.

Peter Kirkpatrick

ORIGINAL RESEARCH PAPER Meister, S. et al. Imaging of Plasmodium liver stages to drive next-generation antimalarial drug discovery. Science 334, 1372-1377 (2011)

FURTHER READING Wu, T.et al. Imidazolopiperazines: hit to lead optimization of new antimalarial agents. J. Med. Chem. 54, 5116-5130 (2011) | Mazier, D. et al. A pre-emptive strike against malaria's stealthy hepatic forms. Nature Rev. Drug Discov. 8, 854-864 (2009) 